\title{
Maestros de la medicina interna chilena
}

\author{
Masters of Internal Medicine, in Chile
}

Master of Internal Medicine is the highest award granted by Sociedad Médica de Santiago-Chilean Society of Internal Medicine. Since this award was instituted (1981), the Society has given it periodically to the most distinguished Chilean physicians. All awardees are considered model physicians, medical educators and individuals, to be emulated by the future generations, with the responsibility of maintaining the spirit, the prestige and the projection of Internal Medicine into the future. In November 2010, on the occasion of the XXXII ${ }^{\text {nd }}$ Chilean Congress of Internal Medicine, the title of "Master of Internal Medicine" was awarded to Rodolfo Armas-Merino, M.D., M.A.C.P. Dr Armas is Professor of Medicine at the University of Chile School of Medicine, Member of the Chilean National Academy of Medicine (currently its President) and Master of the American College of Physicians. The award was presented by Gloria López-Stewart, M.D. and the text of her presentation is included. The following list updates the names of those Chilean physicians that have been awarded this distinction.

(Rev Med Chile 2011; 139: 391-394).

Key words: Awards and Prizes; Internal Medicine; Societies, medical.

"Maestro de la Medicina Interna" es la más alta distinción honorifica otorgada a sus miembros por la Sociedad Médica de Santiago - Sociedad Chilena de Medicina Interna. Desde que fue instituido, en 1981, la Sociedad ha otorgado el título a distinguidos internistas chilenos, en una selección que ocurre periódicamente. Los galardonados son considerados modelos como médicos, educadores y personas, dignos de ser emulados por las futuras generaciones de médicos, con la responsabilidad de mantener el espíritu, prestigio y proyección de la medicina interna. En 2010, en ocasión del XXXII Congreso Chileno de Medicina Interna, se entregó el título de "Maestro de la Medicina Interna» al Dr. Rodolfo Armas Merino. El Dr. Armas es Profesor de Medicina en la Universidad de Chile, Miembro de Número de la Academia Chilena de Medicina (actualmente su Presidente) y "Master" del American College of Physicians. Ha sido un colaborador permanente de la Revista Médica de Chile, como revisor externo y Miembro del Comité Editorial Asesor. En su homenaje se publica la presentación que hizo la Dra. Gloria López Stewart, al entregarle la actual distinción. Una sintesis del curriculum vitae del Dr. Armas y su foto se publicaron en el número de junio de la Revista (Rev Med Chile 2010; 138: 784-5).

La siguiente nómina actualiza la lista de quienes han recibido el título de "Maestro de la Medicina Interna", en Chile:

\begin{tabular}{|ll|}
\hline 1981 & Rodolfo Armas Cruz \\
\hline 1983 & Ramón Valdivieso Delaunay \\
\hline 1985 & Luis Hervé Lelievre \\
\hline 1987 & Francisco Rojas Villegas \\
1989 & Arturo Atria Ramírez \\
\hline 1992 & Mario Plaza De Los Reyes Tapia \\
\hline 1994 & Roque Kraljevic Orlandini \\
\hline 1996 & Fernando Valenzuela Ravest \\
\hline 1998 & Esteban Parrochia Beguin \\
\hline 2000 & Guillermo Ugarte Manterola \\
\hline 2002 & Raúl Etcheverry Barucchi y Camilo Larraín Aguirre \\
\hline 2005 & Alejandro Goic Goic \\
\hline 2007 & Manuel García de los Ríos Álvarez \\
\hline 2010 & Rodolfo Armas Merino \\
\hline
\end{tabular}


Dr. Rodolfo Armas Merino:

\section{Maestro de la Medicina Interna 2010*}

Premiar y agradecer es un acto humano que gratifica tanto al premiado como a quien lo honra. En el momento de hacer una reseña que permita apreciar los méritos de la persona que recibe una distinción, creo importante conocer las cualidades que se están valorando, la historia del Premio, y el proceso que condujo a su elección.

En primer término, es necesario recordar que la Sociedad Médica de Santiago-Sociedad Chilena de Medicina Interna (SMS), es la organización médica más antigua de nuestro país (1869) y constituye el centro y origen de todas las organizaciones actuales. Representa la esencia de los principios societarios, el interés por el progreso y desarrollo de la medicina chilena, de su ejercicio ético y de la salud de la población. Desde su fundación, impulsada por estudiantes de Medicina, sus miembros consideraron fundamental premiar a quienes, entre sus pares, representaban mejor estos ideales, como un gesto de gratitud y reconocimiento y también de demostración para las nuevas generaciones de modelos dignos de seguir e imitar. Fue así como se decidió premiar al "Maestro". Este término implica la maestría y dominio de una disciplina, la capacidad de transmitir esas competencias al más alto nivel posible y la trayectoria de formación de una escuela de discípulos con su sello personal. Fue necesario un largo proceso de consolidación, maduración y crecimiento para que nuestra Sociedad distinguiera a su primer "Maestro de la Medicina", al Profesor Dr. Rodolfo Armas Cruz, padre del Maestro 2010. El selecto grupo de 17 Maestros que lo sucedieron, reúne con diferentes matices estas extraordinarias cualidades. La SMS otorga una importancia trascendente a la selección de candidatos y a la elección, con un proceso regulado, objetivo y serio.

El Dr. Rodolfo Armas Merino, nuestro Maestro de la Medicina 2010, pasa hoy a integrar esta magnífica galería de excelencia.

No resulta fácil sintetizar la obra del Dr. Armas Merino, tan amplio es su campo de realizaciones y tan alto el nivel logrado en todas ellas. Intentaré hacerlo para quienes no han tenido el privilegio de conocerlo personalmente. Para ello, además de su extenso curriculum vitae y la experiencia de haber compartido lugar de trabajo y varias experiencias en directorios de Sociedades, he contado con la generosa colaboración de quienes trabajaron día a día en el Departamento de Gastroenterología del Hospital San Juan de Dios, desde donde proyectó toda su carrera en nuestro país. La Dra. Ximena Morales resumió hermosamente esas vivencias en su discurso de presentación del Dr. Armas Merino durante la ceremonia de entrega del Premio Nacional de Medicina 2010, en el mes de mayo.

El Dr. Armas es especialista en Medicina Interna y Gastroenterología, con formación Universitaria nacional, y hepatólogo con formación en el Reino Unido (Londres), Los Ángeles (EE UU de NA) y México. Desarrolla hasta la actualidad su trabajo en el Hospital San Juan de Dios. Tiene la categoría de Profesor Titular de Medicina de la Universidad de Chile y ha ocupado cargos de alto nivel y responsabilidad académica y de servicio. Ha sido Miembro del Consejo Normativo (197072), Secretario de Estudios (1973-74), Presidente de la Comisión de Docencia (1974-76), y Director del Departamento de Medicina (1986-1994) en la Sede Occidente de la Facultad de Medicina de la Universidad de Chile. Fue Miembro del Consejo Superior de Desarrollo Tecnológico y del Consejo Asesor de CONICYT (1994) y de Comités en el Fondo Nacional de Investigación Científica y Tecnológica (FONDECYT) y de la Comisión Nacional de Acreditación de Pregrado (CONAP).

$\mathrm{Su}$ actividad en sociedades médicas ha sido y es notable: Presidente de la Sociedad Médica de Santiago (1981-83); Presidente de la Comisión $\mathrm{Na}$ cional Autónoma de Certificación de Especialidades Médicas (CONACEM) (1987-1994); Director de la Sociedad Chilena de Gastroenterología (19951990); Gobernador del Capítulo Chileno del American College of Physicians (1996-2000); Presidente fundador de la Asociación de Sociedades Científicas Médicas de Chile (ASOCIMED); Vicepresidente del Fondo Nacional de Investigación en Salud (FONIS), Miembro del Consejo Superior de Desarrollo Tecnológico y del Consejo Asesor de CONICYT (1994) y Secretario de la Academia Chilena de Medicina (2008 a la fecha). En el momento de

*Texto basado en el discurso de presentación del Premio, durante el XXXII Congreso Chileno de Medicina Interna, Viña del Mar, noviembre de 2010. 
esta publicación el Dr. Armas es Presidente de la Academia de Medicina del Instituto de Chile.

Ha sido pionero en Chile en las investigaciones sobre porfiria y ha realizado investigaciones sobre afecciones crónicas no alcohólicas del hígado, con más de cien publicaciones sobre estos temas en revistas nacionales e internacionales.

Ha publicado 4 libros sobre gastroenterología y hepatología: Progresos en Hepatología, Avances en Gastroenterología, Hígado, Vías Biliares y Páncreas, Enfermedades Hepáticas Crónicas No Alcohólicas, y es autor de numerosos capítulos de libros (20).

Es interesante destacar que 26 de sus 130 publicaciones versan sobre temas como formación médica, especialización, conflictos de intereses, investigación en Medicina, la importancia del apoyo al médico joven y otros.

Se ha dado el tiempo para la actividad gremial, siendo miembro del Consejo Regional Santiago (1969-1973) y del Consejo Nacional del Colegio Médico de Chile (1973-1975).

Muy destacable es su participación como integrante de la Comisión Nacional de Reparación y Reconciliación, durante 1992.

Los Premios y distinciones que ha recibido son numerosos: por sus trabajos de investigación, siete distinciones, además del Premio Rodolfo Armas Cruz 1988 de Educación Médica, otorgado por el Colegio Médico de Chile. Fue designado Socio Honorario de la Sociedad Médica de Santiago en1988, Miembro de Número de la Academia Chilena de Medicina en1989, Miembro Honorario de la Sociedad Chilena de Gastroenterología (1998), "Master" del American College of Physicians (2001) y, finalmente, en mayo de 2010 recibió el Premio Nacional de Medicina.

Todo lo señalado es mérito suficiente para recibir la distinción de Maestro de la Medicina. Pero el Dr. Armas Merino reúne muchas otras excepcionales cualidades, algunas de las cuales se reflejan en sus realizaciones innovadoras y otras que hacen que el trabajo en equipo junto a él sea grato y altamente productivo.

Me permitiré enumerar y describir estas singularidades:

1. Capacidad intuitiva, anticipatoria para captar la necesidad o conveniencia de realizar determinadas acciones en bien de las comunidades médicas y de la calidad de la atención del paciente.
2. Inteligencia emocional, que facilita el establecimiento de relaciones cordiales de amistad que conducen a acuerdos que se concretan en acciones. Establecimiento de relaciones sólidas, abiertas. Contactos y amistades en todos los sectores.

3. Dominio extraordinario del trabajo en equipo, multiprofesional y multidisciplinario.

4. Capacidad de trabajo incansable, sin que ello sea percibido. No lo recuerdo con aspecto agotado.

5. Sentido del humor extraordinario.

6. Generosidad a toda prueba.

7. Humildad y sencillez.

8. Amplia gama de intereses culturales, artísticos y sociales. Es el médico más informado que yo personalmente conozca. (Si Ud. desea saber qué está ocurriendo, ocurrirá y por qué, la participación con el Dr. Armas en cualquier comisión, directorio o sociedad, le garantiza una información actualizada).

9. Espíritu familiar, reflejado en la presencia constante de su esposa, hijos, nietos y de sus padres en todas las conversaciones, reuniones de trabajo y en las actividades sociales. Todo en medio de alegría, a pesar de momentos difíciles y de profunda tristeza.

10. Extraordinaria vocación de servicio.

Pienso que lo descrito permite comprender cómo el Dr. Armas ha podido incentivar, catalizar y concretar la fundación de organizaciones tan importantes y pioneras en la realidad médica chilena y latinoamericana, como CONACEM, que en sus 28 años de vida ha certificado a miles de especialistas médicos, ASOCIMED, que reúne organizadamente a las Sociedades Científicas Médicas de Chile, la cual se ha incorporado activamente al proceso de acreditación de Programas de Formación de Especialistas médicos, por intermedio de la Agencia APICE, la creación del Fondo para la Investigación en Salud, que ha significado un impulso para la investigación con repercusión en Salud Pública. Otras iniciativas están en proceso y ya sea que el propio Dr. Armas las implemente o por la evidencia de su necesidad revelada por él, lleven o hayan llevado a las autoridades a su creación: certificación de calidad de los Servicios, prevención de errores en Medicina, regulación de conflictos de intereses y relaciones con las empresas farmacéuticas y de productos médicos y muchos otros temas. 
Termino expresando que pocas veces es tan grato presentar a un premiado como en esta ocasión. He tenido el privilegio de trabajar como su colaboradora en el Departamento de Medicina Occidente, hace muchos años, como vecina en el edificio de la Unidad de Diabetes del Hospital San Juan de Dios, hace menos tiempo y como Secretaria en ASOCIMED y Miembro de la Academia Chilena de Medicina, en los últimos años. Debo señalar que siempre he aprendido con él a pensar crítica y a la vez objetivamente, con una visión fresca; a trabajar organizadamente y sin aspavientos en pro del progreso de la Medicina chilena. El fruto de su trabajo ha surgido con esfuerzo y alegría, entre risas y sonrisas, obteniendo lo mejor de cada uno de sus colaboradores. Me asiste la certeza de que el listado de médicos, profesionales de la salud, funcionarios administrativos que le deben a nuestro flamante “Maestro de la Medicina 2010" legados similares, es casi interminable. Esta nueva distinción muestra la gratitud de la medicina, los médicos y de la población chilena que se ha beneficiado con su gran trabajo.

Gloria López S.

Profesora Adjunta de Medicina, Facultad de Medicina de la Universidad de Chile. Expresidente de la Sociedad Médica de Santiago-Sociedad Chilena de Medicina Interna. Académica de Número, Academia Chilena de Medicina. 